\title{
Robust quantum gates on neutral atoms with cavity-assisted photon scattering
}

\author{
L.-M. Duan, ${ }^{1}$ B. Wang, ${ }^{1}$ and H. J. Kimble ${ }^{2}$ \\ ${ }^{1}$ FOCUS Center and MCTP, Department of Physics, University of Michigan, Ann Arbor, Michigan 48109-1120, USA \\ ${ }^{2}$ Norman Bridge Laboratory of Physics 12-33, California Institute of Technology, Pasadena, California 91125, USA
}

(Received 6 July 2005; published 28 September 2005)

\begin{abstract}
We propose a scheme to achieve quantum computation with neutral atoms whose interactions are catalyzed by single photons. Conditional quantum gates, including an $\mathrm{N}$-atom Toffoli gate and nonlocal gates on remote atoms, are obtained through cavity-assisted photon scattering in a manner that is robust to random variation in the atom-photon coupling rate and which does not require localization in the Lamb-Dicke regime. The dominant noise in our scheme is automatically detected for each gate operation, leading to signalled errors which do not preclude efficient quantum computation even if the error probability is close to the unity.
\end{abstract}

DOI: 10.1103/PhysRevA.72.032333

PACS number(s): 03.67.Lx, 03.67.Hk, 42.50.Pq

Neutral atoms in optical cavities have been one of the pioneering avenues for the implementation of quantum computation and networking [1-4]. Nevertheless, the experimental requirements associated with these approaches turn out to be very challenging. In particular, although significant experimental advances have been reported recently in transmitting and trapping single atoms in high finesse cavities [4-12], no experiment has yet achieved a well defined number of atoms $N \geqslant 2$ each of which is strongly coupled to the cavity mode, individually addressable, and localized to the Lamb-Dicke limit, as is required for the protocol of Ref. [1]. To realize a more scalable system, Chapman et al. proposed an architecture in which a transverse optical lattice is employed to translate atoms into and out of a high-finesse cavity for entangling gate operations [6]. Transport that preserves internal state coherence has been demonstrated for both ions [13] and atoms [14]. However, although the approach of Ref. [6] does solve the problem of separate addressing of many atoms in a tiny cavity, there remain significant obstacles to achieving Lamb-Dicke confinement [15] and strong coupling for any scheme that has yet been proposed.

To overcome these difficulties and to provide several capabilities for quantum logic, in this paper we propose a scheme for atomic quantum gates whereby atom-atom interactions are catalyzed by single photons in a fashion that is robust to various sources of practical noise. More specifically, a controlled phase-flip gate between two atoms is achieved by cavity-assisted scattering of a single-photon pulse from the cavity in which the atoms are localized [16]. This gate is insensitive to uncertainties in the atom-photon coupling rate, thereby obviating the requirement for LambDicke localization. It is also robust to all sources of photon loss, including, for instance, atomic spontaneous emission, photon collection and detection inefficiency, and any vacuum component in the scattering pulse. Such noise is automatically detected for each gate, leading to a finite failure probability of the gate operation. As shown in Refs. [17,18], efficient quantum computation can nevertheless be achieved even if the associated failure probability is close to unity. Moreover, our scheme can be readily extended to achieve a Toffoli gate for $N$ atoms in a single step and to realize nonlocal gates on remote atoms trapped in different cavities. The direct $N$-bit gate could lead to more efficient construction of quantum circuits, and the nonlocal gates on remote atoms naturally integrates local computation with quantum networking.

To explain the idea of the gate operation, we first consider two atoms in a single-sided cavity. To have a scalable architecture, one can follow Ref. [6] to assume there are transverse optical lattice potentials to move the target atoms into and outside the cavity $[19,20]$. Each atom has three relevant levels as shown in Fig. 1. The qubit is represented by different hyperfine levels $|0\rangle$ and $|1\rangle$ in the ground-state manifold. The atomic transition from $|1\rangle$ to an excited level $|e\rangle$ is resonantly coupled to a cavity mode $a_{c}$. The state $|0\rangle$ is decoupled due to the large hyperfine splitting.

To perform a collective quantum gate on the two atoms, we reflect a single-photon pulse from the cavity. This singlephoton pulse, with its state denoted as $|p\rangle$, is resonant with the bare cavity mode $a_{c}$. If the photon pulse is sufficiently long (with its bandwidth $\Delta \Omega$ much smaller than the cavity decay rate $\kappa$ ), reflection of the pulse from a resonant cavity absent an atom will leave the pulse shape almost unchanged but will flip its global phase, as we later characterize in detail. For the case that both of the atoms are in the $|0\rangle$ state, this is precisely the nature of the resonant reflection since

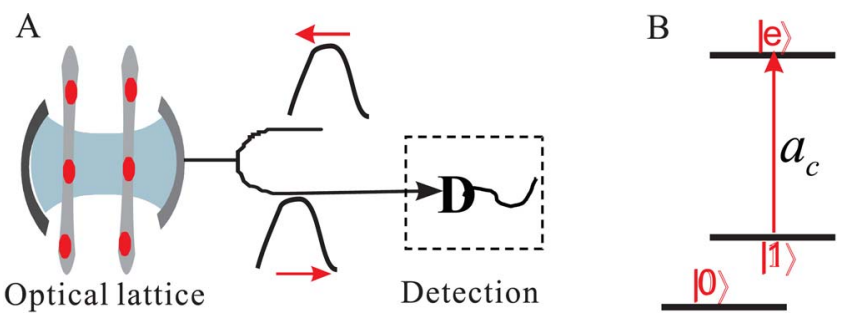

FIG. 1. (Color online) (A) Schematic setup for implementation of the controlled phase flip ( $\mathrm{CPF}$ ) gate on two atoms inside the cavity through the photon-scattering interaction. Any pair of atoms can be transmitted into the cavity for a collective gate operation through a transverse optical lattice potential as suggested in Refs. $[6,21]$. For a more robust implementation of the gate, we add a single-photon detector to detect the output photon pulse as illustrated inside the dashed box. (B) The relevant level structure of the atoms and the coupling configuration. 
there is negligible atom-cavity coupling and hence no shift of the resonant frequency of the cavity mode. After reflection, the atom-photon state $|0\rangle_{1}|0\rangle_{2}|p\rangle$ evolves into $-|0\rangle_{1}|0\rangle_{2}|p\rangle$, where the subscripts 1,2 denote the two intracavity atoms. However, if either or both of the atoms are in the state $|1\rangle$, the effective frequency of the dressed cavity mode will be shifted due to the atom-cavity coupling, which is described by the Hamiltonian

$$
H=\hbar \sum_{i=1,2} g_{i}\left(|e\rangle_{i}\left\langle 1\left|a_{c}+\right| 1\right\rangle_{i}\langle e| a_{c}^{\dagger}\right) .
$$

If the coupling rates $g_{i} \gg\left(\Delta \Omega, \kappa, \gamma_{s}\right)$, where $\gamma_{s}$ is the rate of spontaneous decay of $|e\rangle$, then the frequency shift will have a magnitude comparable with $g_{i}$, so that the incident singlephoton pulse will be reflected by an off-resonant cavity. Hence, both the shape and global phase will remain unchanged for the reflected pulse. Due to this property, the component states $|0\rangle_{1}|1\rangle_{2}|p\rangle,|1\rangle_{1}|0\rangle_{2}|p\rangle$, and $|1\rangle_{1}|1\rangle_{2}|p\rangle$ are likewise unaffected by reflection process. The net effect of these two subprocesses is that the reflection of a singlephoton pulse from the cavity actually performs a controlled phase-flip gate (CPF) $U_{12}=\exp \left(i \pi|00\rangle_{12}\langle 00|\right)$ on the two atoms while leaving the photon state unchanged (unentangled). Hence, in the ideal case the reflected photon can be utilized to catalyze subsequent gate operations.

However, in a realistic setting our scheme can be performed in a more robust fashion by detecting the output pulse with a single-photon detector. By this means, gate errors due to all sources of photon loss, including atomic spontaneous emission, cavity mirror absorption and scattering, imperfection in the photon source, and photon collection and detection inefficiencies, are always signaled by the absence of a photon count. As a result, these dominant sources of noise only lead to probabilistic signaled errors, which yield a finite failure probability of the gate but which have no contribution to the gate infidelity if the operation succeeds (i.e., if a photon count is registered). For this class of errors, efficient quantum computation is possible with an arbitrarily small gate success probability $p$ [17]. Compared with deterministic gates, the required extra computational overhead due to the small gate success probability $p$ scales efficiently (polynomially) both with $1 / p$ and the computational scale characterized by the number of qubits $n$ [17]. Because of this robustness, the input single-photon pulse can also be replaced by a simple weak coherent pulse $|\alpha\rangle$ with the mean photon number $|\alpha|^{2} \ll 1$. This replacement does not give any essential problem in terms of scaling, although the individual gate efficiency (the success probability) is indeed significantly reduced by a factor of $|\alpha|^{2}$.

Before going to the detailed theoretical characterization of the gate fidelity and efficiency, we next present some extensions of the above scheme. First, our scheme can be readily extended to perform a Toffoli gate on $N$ atoms in a single time step. If one reflects a single-photon pulse from a cavity with $N$ atoms trapped inside, the pulse will have a flip of its global phase if and only if all the atoms are in the $|0\rangle$ state. So, this reflection performs a Toffoli gate $U_{12 \cdots N}$ $=\exp \left(i \pi|00 \cdots 0\rangle_{12 \cdots N}\langle 00 \cdots 0|\right)$ on all the atoms while leaving the photon state unentangled. This direct $N$-bit gate could

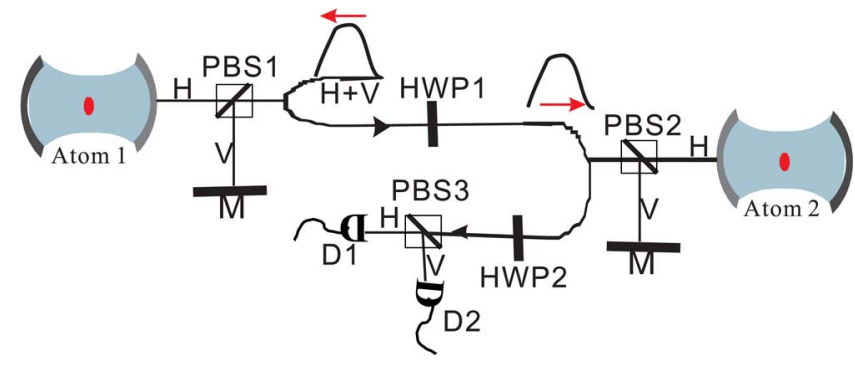

FIG. 2. (Color online) Schematic of the setup for implementation of nonlocal gates on two atoms 1 and 2 trapped in distant cavities. Not shown are circulators (e.g., Faraday devices) to redirect the output beams along paths distinct from the inputs. See the text for further explanation.

lead to more efficient construction of circuits for quantum computation. For instance, the reflection operation in the Grover's search algorithm can be realized in a single step with the $N$-bit Toffoli gate [21].

Second, the above scheme can also be extended to perform nonlocal gates on two remote atoms trapped in different cavities, as illustrated in Fig. 2. A similar nonlocal gate was also proposed recently in a different system with rare-earth atoms [22]. For this purpose, one uses a single-photon (or weak-coherent) pulse which is in an equal superposition state $(|H\rangle+|V\rangle) / \sqrt{2}$ of the $H$ and $V$ polarization components. With a polarization beam splitter (PBS1), the $H$ and $V$ components of the pulse are "bounced" back from the atom-cavity system and a mirror $M$, respectively, with the reflection from $M$ leaving the incident pulse unchanged. The overall reflection from the cavity and the mirror $M$ actually performs the gate operation $U_{1 p}=\exp \left(i \pi|0 H\rangle_{1 p}\langle 0 H|\right)$ on atom 1 and the photon pulse $p$, so that there is a phase flip only when the atom is in the state $|0\rangle$ and the photon is in the polarization $|H\rangle[16]$. The pulse is reflected successively from the two cavity setups, with a half-wave plate (HWP1) inserted into the optical path between the two reflections which performs a Hardmard rotation on the photon's polarization $|H\rangle \rightarrow(|H\rangle$ $+|V\rangle) / \sqrt{2},|V\rangle \rightarrow(|V\rangle-|H\rangle) / \sqrt{2}$. The photon is detected by two single-photon detectors D1 and D2 after the reflections, corresponding to a measurement of its polarization in the basis $\left(|V\rangle_{ \pm}|H\rangle\right) / \sqrt{2}$ (after the HWP2 and the PBS3; see Fig. 2). For a detection event in $\mathrm{D} 2$, a phase flip operation $\sigma_{1}^{z}$ is performed on the atom 1, while no operation is applied if D1 clicks. The net effect of these operations is the desired CPF gate $U_{12}=\exp \left(i \pi|00\rangle_{12}\langle 00|\right)$ on the two remote atoms 1,2 . Among other applications, this nonlocal gate and its extension to multiple atom-cavity systems provide a convenient avenue for quantum networking. As before for the case of a single cavity, in this distributed setting any noise leading to photon loss is always signaled by the absence of a photon count from either D1 or D2.

We now present a more detailed theoretical model of our scheme and characterize the influence of some practical sources of noise. The input single-photon pulse with a normalized shape function $f_{\text {in }}(t)$ and a duration $T$ can be described by the state $|p\rangle=\int_{0}^{T} f_{\text {in }}(t) a_{\mathrm{in}}^{\dagger}(t) d t|\mathrm{vac}\rangle$, where $|\mathrm{vac}\rangle$ denotes the vacuum state and $a_{\mathrm{in}}^{\dagger}(t)$ is the one-dimensional 
optical field operator with the commutation relation $\left[a_{\mathrm{in}}(t), a_{\mathrm{in}}^{\dagger}\left(t^{\prime}\right)\right]=\delta\left(t-t^{\prime}\right)[23]$. The cavity mode $a_{c}$ is driven by the input field $a_{\text {in }}(t)$ through the Langevin equation [23]

$$
\dot{a}_{c}=-i\left[a_{c}, H\right]-(\kappa / 2) a_{c}-\sqrt{\kappa} a_{\mathrm{in}}(t),
$$

where $\kappa$ is the cavity decay rate and the Hamiltonian $H$ is given in Eq. (1) for the case of two atoms; generalization to multiple atoms is straightforward. To account for atomic spontaneous emission with a rate $\gamma_{s}$, we add an effective term $\left(-i \gamma_{s}\right)|e\rangle\langle e|$ to the Hamiltonian $H$. The output field $a_{\text {out }}(t)$ of the cavity is connected with the input through the input-output relation $a_{\text {out }}(t)=a_{\text {in }}(t)+\sqrt{\kappa} a_{c}$.

The final atom-photon state can be numerically solved from the above set of equations through discretization of the continuum optical fields (for details on the numerical method, see Refs. [16,24]). We use the following two quantities to characterize the imperfections in our scheme. (1) Due to various sources of photon loss, photons in the cavity may be lost with then no photon count at the detectors. Hence, we calculate the success probability of a photon count at the detector to characterize the efficiency of the scheme. (2) Even if a photon emerges, there may still be imperfections of the atomic gate mainly due to the shape distortion of the photon pulse after reflection from the cavity, which can be characterized through the gate fidelity. Without loss of the photon, the final atom-photon state can be written as $\left|\Psi_{\text {out }}\right\rangle=\sum_{i_{1} i_{2}} c_{i_{1} i_{2}}\left|i_{1} i_{2}\right\rangle_{a}|p\rangle_{i_{1} i_{2}}$, where $\sum_{i_{1} i_{2}} c_{i_{1} i_{2}}\left|i_{1} i_{2}\right\rangle_{a}\left(i_{1}, i_{2}\right.$ $=0,1)$ is the general form for the input state of the two atoms. The output photon state $|p\rangle_{i_{1} i_{2}}$ corresponds to the atomic component $\left|i_{1} i_{2}\right\rangle_{a}$, and is given by $|p\rangle_{i_{1} i_{2}}$ $=\int_{0}^{T} f_{i_{1} i_{2}}^{\text {out }}(t) a_{\text {out }}^{\dagger}(t) d t|\mathrm{vac}\rangle$ with a shape $f_{i_{1} i_{2}}^{\text {out }}(t)$. Ideally, the output state $\left|\Psi_{\text {out }}^{\text {id }}\right\rangle$ would have the shape functions $f_{00}^{\text {out }}(t)=$ $-f_{\text {in }}(t)$ and $f_{i_{1} i_{2}}^{\text {out }}(t)=f_{\text {in }}(t)$ (for $i_{1}, i_{2} \neq 0$ ), which realizes a perfect $\mathrm{CPF}$ gate $U_{12}$ on the atoms. Hence to characterize the gate imperfection, we calculate the fidelity $F$ $\equiv\left|\left\langle\Psi_{\text {out }}^{\text {id }} \mid \Psi_{\text {out }}\right\rangle\right|^{2}$, which is directly extendable to any number of atoms. In the following calculation of the fidelity $F$, we choose the input state $[(|0\rangle+|1\rangle) / \sqrt{2}]^{\otimes N}$ for the case of $N$ atoms.

The results from our calculations are summarized in Fig. 3. First, Fig. 3(a) shows the component pulse shape $f_{i_{1} i_{2}}^{\text {out }}(t)$ corresponding to a Gaussian input $f_{\text {in }}(t)$ for the case of two atoms. Only the component $f_{00}^{\text {out }}(t)$ has a notable shape distortion; all others are basically indistinguishable from the input. To account for random variation in the coupling rates $g_{i}$, we have also calculated $f_{i_{1} i_{2}}^{\text {out }}(t)$ for $g_{i}$ varying from $2 \kappa$ to $6 \kappa$. The output pulse shapes are nearly identical for $g_{i}$ varying in this range, which is typical of current experiments [4-10]. Figure 3(b) shows the corresponding fidelity $F$ of the CPF (or Toffoli) gate from the shape distortion noise with the atom number $N=2,3,4,5$. The fidelity $F$ improves with increase of the pulse duration $T$ since the shape distortion is reduced for longer pulses. $F$ also increases with the atom number $N$, which is a bit surprising but actually reasonable: for the $N$-atom state $[(|0\rangle+|1\rangle) / \sqrt{2}]^{\otimes N}$, the fraction of the component $|0\rangle^{\otimes N}$ goes down as $1 / 2^{N}$, and the pulse shape distortion noise comes dominantly from this component. Be-
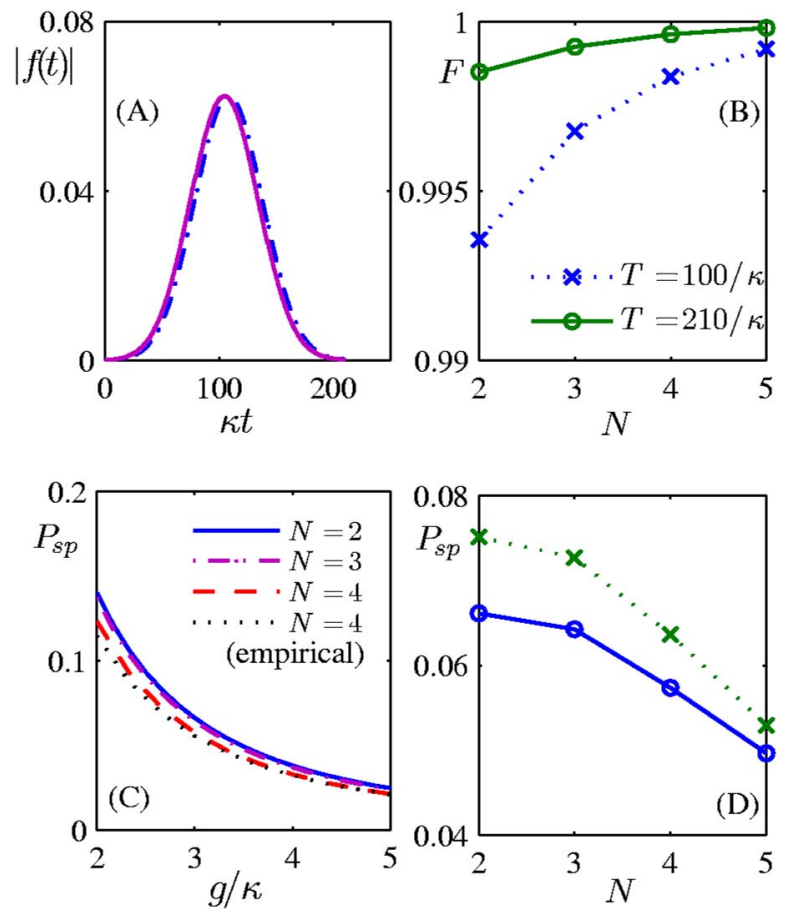

FIG. 3. (Color online) (A) The shape functions $|f(t)|$ for the input pulse (solid curve) and the reflected pulses with the atoms in different component states $\left|i_{1} i_{2}\right\rangle_{a}$. The shape function for the atom in the state $|00\rangle_{a}$ is shown by the dash-dot curve. With the coupling rate $g$ in a typical range from $2 \kappa$ to $6 \kappa$, the shape functions for the atoms in all the other component states are indistinguishable from that of the input pulse (the solid curve). We have assumed a Gaussian shape for the input pulse with $f_{\text {in }}(t) \propto \exp \left[-(t-T / 2)^{2} /(T / 5)^{2}\right]$, where $t$ ranges from 0 to $T$ and $T=210 / \kappa$ for this example. (B) The gate fidelity versus the number of atoms with the pulse duration $T$ $=100 / \kappa$ (the dotted curve) and $T=210 / \kappa$ (the solid curve), respectively. (C) The photon loss probability $P_{\text {sp }}$ due to atomic spontaneous emission shown as a function of the coupling rate $g$ in units of $\kappa$ with the atom number $N=2,3,4$. The dotted curves shows $P_{\mathrm{sp}}$ calculated from the empirical formula given in the text for $N=4$. (D) Comparison of the photon loss $P_{\mathrm{sp}}$ for a constant coupling rate $g=3 \kappa$ (the solid curve) and for a time varying rate $g_{i}(t)=3 \kappa[1$ $\left.+\sin \left(\nu t+\phi_{i}\right) / 3\right]$ (the dotted curve) for the ith atom, where $\nu=\kappa / 6$ corresponds to a typical atom's axial oscillation frequency in the trap, and $\phi_{i}$ are taken as random numbers accounting for the atoms' random initial positions. $g_{i}(t)$ is chosen so that its maximum and minimum differ by a factor of 2 , which exceeds that in current experiments [9]. Other parameters for (A) and (B) are $\gamma_{s}=\kappa$ and $g=3 \kappa$, and for (C) and (D), $\gamma_{s}=\kappa$ and $T=210 \kappa$.

cause the component $|0\rangle^{\otimes N}$ dominates the contribution to the gate infidelity, $F$ is also very insensitive to variation of the coupling rates $g_{i}$. We have verified that there is no notable change of $F\left(\delta F<10^{-4}\right)$ in Fig. 3(b) for $g_{i}$ varying from $2 \kappa$ to $6 \kappa$.

Any source of photon loss has no contribution to the gate fidelity but instead influences gate efficiency (success probability). A fundamental source of photon loss is atomic spontaneous emission. Figure 3(c) shows the failure probability $P_{\text {sp }}$ of the gate due to this source of noise, with the noise rate $\gamma_{s}=\kappa$. For $N$ atoms with equal $g_{i}=g$, the probability $P_{\mathrm{sp}}$ can be well fit by an empirical formula $P_{\mathrm{sp}} \approx P_{\mathrm{emp}}$ 
$\equiv \sum_{n=1}^{N}\left[N ! / n !(N-n) ! 2^{N}\right]\left[1+n g^{2} / \kappa \gamma_{s}\right]^{-1}$. The empirical $P_{\text {emp }}$ can be understood as a probability averaged over all the Dicke-state components in the input state $[(|0\rangle+|1\rangle) / \sqrt{2}]^{\otimes N}$, with the $n$th Dicke-component having an effective coupling rate $\sqrt{n} g$ to the cavity mode. We have also simulated the loss probability $P_{\mathrm{sp}}$ when the coupling rates $g_{i}$ are different and vary during the gate operation, for instance, as would be caused by the atoms' thermal motion. With some typical choice of the relevant experimental parameters, the result is shown in Figure $3 \mathrm{D}$, which is qualitatively similar to the constant coupling rate case with an effective average over $\left|g_{i}\right|$. Other sources of photon loss can be similarly characterized. For instance, with a finite photon collection and detection efficiency $\eta$, the success probability of each gate will be simply reduced by a factor of $\eta$.

In summary, we have proposed a new scheme for robust atomic gates by way of interactions mediated by cavityassisted photon scattering. These gates are robust to all sources of photon loss that are typically the dominant source of noise in experimental implementations, and are furthermore insensitive to randomness in the coupling rates caused by fluctuations in atomic position. Beyond two-atom gates illustrated in Fig. 1, our scheme can also be employed for realization of an $\mathrm{N}$-atom Toffoli gate in a single step and for the implementation of nonlocal gates on distant atoms as in Fig. 2. We have characterized the efficacy of our scheme through exact numerical simulations that incorporate various sources of experimental noise. These results demonstrate the practicality of our scheme by way of current experimental technology.

Note added. After submission of this work, we were informed that a similar idea was also investigated by the authors X.-M. Lin et al. (unpublished).

We gratefully acknowledge the contributions of W. D. Phillips, whose question stimulated these investigations. We thank also S. Lloyd for helpful discussions. This work was supported by the National Science Foundation (Grant Nos. 0431476, EIA-0086038, and PHY-0140355), the Advanced Research and Development Activity under ARO contracts, the A. P. Sloan foundation (L.M.D.), and the Caltech MURI Center for Quantum Networks (H.J.K.).
[1] T. Pellizzari, S. A. Gardiner, J. I. Cirac, and P. Zoller, Phys. Rev. Lett. 75, 3788 (1995).

[2] Q. A. Turchette et al., Phys. Rev. Lett. 75, 4710 (1995).

[3] J. I. Cirac, P. Zoller, H. J. Kimble, and H. Mabuchi, Phys. Rev. Lett. 78, 3221 (1997).

[4] For a review, see C. Monroe, Nature (London) 416, 238 (2002)

[5] J. McKeever et al., Phys. Rev. Lett. 90, 133602 (2003).

[6] M. Chapmann, unpublished; J. A. Sauer et al., Phys. Rev. A 69, 051804(R) (2004).

[7] P. Maunz et al., Nature (London) 428, 50 (2004).

[8] J. McKeever, J. R. Buck, A. D. Boozer, and H. J. Kimble, Phys. Rev. Lett. 93, 143601 (2004).

[9] A. Boca et al., Phys. Rev. Lett. 93, 233603 (2004).

[10] P. Maunz et al., Phys. Rev. Lett. 94, 033002 (2005).

[11] M. Keller et al., Nature (London) 431, 1075 (2004).

[12] A. Kreuter et al., Phys. Rev. Lett. 92, 203002 (2004).

[13] M. A. Rowe et al., Quantum Inf. Comput. 2, 257 (2002).

[14] S. Kuhr et al., Phys. Rev. Lett. 91, 213002 (2003).

[15] For example, if a far-off resonance dipole-force trap (FORT) is utilized for conveying an atom along a direction transverse to the cavity axis $[6,14]$ and if an atom is cooled to the motional ground state of the FORT, the spread of the atomic wave packet along the direction of the cavity axis is typically still comparable to the optical wavelength for the cavity QED field due to focusing the FORT beams within the constraints of the cavity geometry. This causes significant uncertainty in the atom-photon coupling rate.

[16] L.-M. Duan and H. J. Kimble, Phys. Rev. Lett. 92, 127902 (2004); B. Wang and L.-M. Duan, Phys. Rev. A 72, 022320 (2005).

[17] L.-M. Duan and R. Raussendorf, Phys. Rev. Lett. 95, 080503 (2005).

[18] S. D. Barrett, and P. Kok, Phys. Rev. A 71, 060310(R) (2005).

[19] The scheme also applies to the more traditional cavity quantum computation architecture with an array of atoms in the cavity. Separate addressing of different atoms could be achieved through frequency selection either with Stark shifts from focused laser beams or with gradients from effective magnetic fields [20].

[20] D. Schrader et al., Phys. Rev. Lett. 93, 150501 (2004).

[21] M. A. Nielsen, I. L. Chuang, Quantum Computation and Quantum Information (Cambridge University Press, Cambridge, 2000).

[22] Yun-Feng Xiao et al., Phys. Rev. A 70, 042314 (2004).

[23] D. F. Walls and G. J. Milburn, Quantum Optics (SpringerVerlag, Berlin, 1994).

[24] L.-M. Duan, A. Kuzmich, and H. J. Kimble, Phys. Rev. A 67, 032305 (2003). 